(1)

CrossMark

\title{
Pseudomonas and risk factor mitigation for chronic lung allograft dysfunction
}

\author{
Allan R. Glanville (1) \\ Affiliation: The Lung Transplant Unit, St. Vincent's Hospital, Sydney, Australia.
}

Correspondence: Allan R. Glanville, The Lung Transplant Unit, St. Vincent's Hospital, Sydney, NSW, Australia 2010. E-mail: allan.glanvilledsvha.org.au

@ERSpublications

Univariable analysis supports attempts at "eradicating" Pseudomonas from bronchopulmonary secretions after lung transplantation https://bit.ly/2Nw6h5P

Cite this article as: Glanville AR. Pseudomonas and risk factor mitigation for chronic lung allograft dysfunction. Eur Respir J 2020; 56: 2001968 [https://doi.org/10.1183/13993003.01968-2020].

The relationship between bronchopulmonary Pseudomonas aeruginosa and chronic lung allograft dysfunction (CLAD) has often stimulated considerations of primacy. Which came first, the chicken or the egg? Unlike binary logic, natural circumstances are more complex and demand a pluralistic view of causality, notwithstanding the recognition that in systems of coexistence, mitigation of deleterious outcomes can still be achieved by reducing one of the driving factors in the equation. So it is with airway disease, both large and small, after lung transplantation $[1,2]$. Mucosal and structural damage promote an unhealthy local milieu with impaired barrier function, compromised local immunity and reduced efficacy of mucociliary transport. Perturbations of the pulmonary microbiome along with chemical damage from often occult gastric aspiration set the foundation for such a scenario. However, unlike fibrotic luminal occlusion of small airways typical of obliterative bronchiolitis, which is permanent, the damage described above may be amenable to change [3]. Of course, in a parallel causation model, the damage described may well alter the local airway environment to facilitate the processes of airway restructuring and, ultimately, loss. So, with this conceptual overview, the attempt by the team from Leuven to "eradicate" airway Pseudomonas can be seen as a potentially worthwhile endeavour, but does it work and are the claims robust [4]? Let us examine the factors critical to the claims. Firstly, the claim of eradication of Pseudomonas is based on examination of airway secretions, sputa and, where available, from scheduled and clinically mandated bronchoalveolar lavage (BAL) fluid culture. Clearly, the sensitivity of these different tools varies greatly and the true negative predictive value is unknown, but clinical experience cautions enthusiasm regarding the ability of eradicating a lower airway pathogen, particularly when biofilms and structurally abnormal airways coincide to protect microdeposits of infection. That is not to say that reducing the colonising load, or perhaps more accurately, the infecting load of Pseudomonas is not a worthwhile goal as outlined above. Indeed, whether Pseudomonas is ever a benign colonising organism in the transplanted lung is open to serious debate, which promotes a genuine attitude that an attempt at eradication is worthwhile provided the therapy is not worse than the disease! A long-term risk/ benefit view is mandated especially as anti-Pseudomonas therapies risk development of more resistant organisms leading to attempts at less effective and more expensive therapies [5]. Similarly, and given that most Pseudomonas infection is hospital acquired, a case may be made for reducing hospital exposure and invasive surveillance procedures unless mandated for specific diagnostic purposes $[6,7]$. Secondly, the time interval to determine success of eradication was necessarily arbitrary (6 months) and the therapies employed were not standardised over time, reflecting one of the common limitations of single-centre 
retrospective studies. Of interest, 23/76 subjects in the successful eradication group did not appear to undergo specific therapies and, conversely, none of the unsuccessful group had a course of quinolone therapy, as described in the supplementary data (table S2 [4]). As interesting as this may be from a pathophysiological perspective, it does not change the ultimate conclusion that patients in whom the "presence" of Pseudomonas could not be detected on respiratory samples had a longer CLAD-free survival and graft survival. Noting this point, it is worth remembering that many registry reports undercount the frequency of CLAD and overcount the CLAD-free period post-transplant, as recently reported by KULKARNI et al. [8]. Most lung transplant recipients die or develop bronchiolitis obliterans syndrome (BOS), as the obstructive phenotype CLAD was termed prior to the current appellation, within 4 years $[9,10]$. Very few remain alive and free from BOS at 10 years [11].

Thirdly, given the multiple risk factors for CLAD, including acute cellular rejection and antibody mediated rejection, do we accept the proposition that Pseudomonas is indeed an independent risk factor for CLAD rather than just a marker of damaged airways $[1,2,12]$ ? Once again, the evidence is largely based on single-centre retrospective studies with some illuminating scientific insights into mechanisms. Вотна et al. [13] in 2008 concluded from a study of 155 consecutive lung transplant subjects that de novo colonisation of the lung allograft by Pseudomonas was strongly associated with the subsequent development of BOS within 2 years of transplant $(23.4 \%$ versus $7.7 \%$ colonised versus not colonised, $\mathrm{p}=0.006)$. Work from VALENTINE et al. [14] in 2009 was in accord but emphasised the importance of pneumonia as a harbinger of BOS. MAINZ et al. [15], in 2012, demonstrated identical $P$. aeruginosa genotypes in the upper airways of patients with cystic fibrosis (CF) prior to and after lung transplant, underlining the risk of upper respiratory tract as a source for colonisation of the transplanted lungs. In support of these findings, BEAUme et al. [16] in 2017 showed that Pseudomonas invaded the host microbiota within 3 days of transplant, with a reduction in richness and diversity of species. Despite antibiotic therapy, Pseudomonas dominated the allograft microbiota until postoperative day 95, perhaps augmented by phenotypic adaptation resulting in increased biofilm formation and swim motility capacity.

BoRTHWicK et al. [17], in a 2011 study of primary bronchial epithelial cells isolated from lung transplant recipients, provided evidence that $P$. aeruginosa, via the activation of monocytic cells, could accentuate transforming growth factor- $\beta 1$-driven epithelial-to-mesenchymal transition which has been implicated in dysregulated epithelial wound repair that contributes to obliterative bronchiolitis after lung transplantation. Using a Cox semi-Markovian approach, Gregson et al. [18] in 2013, showed that the likelihood of transition from transplant to BOS was increased by acute rejection, chemokine ligand (CXCL)5, and the interaction between Pseudomonas and CXCL1. In this study, the Pseudomonas effect was due to infection rather than colonisation. Evidence challenging the benefit of clearance of Pseudomonas has been demonstrated in the CF population by BURKETT et al. [19] in 2012, who showed that declines in lung function, rates of pulmonary exacerbations, deaths or lung transplant were not different between patients who cleared Pseudomonas compared to those who remained persistently infected. Indeed, no clinical benefit was identified in patients who cleared Pseudomonas from sputum compared to those who remained persistently infected. The critical difference likely resides in the innate immune response to the allograft (acute and chronic rejection) versus the response of native airways to infection.

New techniques are now available to map all components of the human pulmonary microbiome and are beginning to inform our understanding [20]. In studies of the microbiome after lung transplant, DiCKSON et al. [21] in 2014 discovered that a distinct species, $P$. fluorescens, was often identified in asymptomatic transplant subjects by pyrosequencing but not detected via the standard bacterial cultures used in the current study, having unique genomic and microbiologic features and widely divergent clinical associations from P. aeruginosa. Following on from these studies, BERNASCONI et al. [22] in 2016 showed that "inflammatory" and "remodelling" profiles were linked to bacterial dysbiosis through mechanistic assays in macrophages, whereas a balanced microbial community maintained homeostasis. In the same year, Borthwick et al. [23] measured alarmins in serial BAL samples and showed that interleukin-1 $\alpha$ was increased in BAL samples of lung transplant recipients growing $P$. aeruginosa and was significantly elevated within 3 months of developing BOS. In short, the case for the risk of $P$. aeruginosa after lung transplantation is well made, but evidence for the benefits of attempts at "eradication", or indeed a reduction in microbial load, is not overwhelming. Hence the current study provides most useful information to guide practice. A number of questions remain, including the utility of aerosolised therapies, which were not employed in this study as they are not reimbursed in Belgium after lung transplantation, despite their use in CF patients to reduce microbial load and hence frequency and severity of acute exacerbations [24]. Also, whether concurrent antibiotic therapy at the time of sample acquisition may have impacted results is not explicitly stated but given the small number of patients in whom Pseudomonas was not "eradicated" likely would have an insignificant biasing effect. The authors have attempted to resolve the matter of retrospective assignation of groups for Kaplan-Meier analysis by using a segmented 
time-based approach (figure 4 [4]), where the benefits of a Pseudomonas-free respiratory tract appear obvious but the numbers are small ( $4-8$ per annum) which serves to magnify the effect. The proper handling of multiple time-dependent effects after lung transplant remains somewhat problematic.

In summary, the goal of eradication of Pseudomonas after lung transplant, as a strategy to reduce the devastating impact of CLAD, now has a building evidence base but as ever, larger, prospective, multicentre studies examining multivariate risks and benefits as well as cost-utility and other strategies to mitigate CLAD are needed, without which the sterling work of these investigators may not be fully appreciated as anything more than the brave beating of a distant drum.

Conflict of interest: A.R. Glanville has nothing to disclose.

\section{References}

1 Glanville AR, Aboyoun CL, Havryk A, et al. Severity of lymphocytic bronchiolitis predicts long-term outcome after lung transplantation. Am J Respir Crit Care Med 2008; 177: 1033-1040.

2 Meyer KC, Raghu G, Verleden GM, et al. An international ISHLT/ATS/ERS clinical practice guideline: diagnosis and management of bronchiolitis obliterans syndrome. Eur Respir J 2014; 44: 1479-1503.

3 Stewart S, Fishbein MC, Snell GI, et al. Revision of the 1996 working formulation for the standardization of nomenclature in the diagnosis of lung rejection. J Heart Lung Transplant 2007; 26: 1229-1242.

4 De Muynck B, Van Herck A, Sacreas A, et al. Successful Pseudomonas aeruginosa eradication improves outcomes after lung transplantation: a retrospective cohort analysis. Eur Respir J 2020; 56: 2001720.

5 Voor In 't Holt AF, Severin JA, Lesaffre EM, et al. A systematic review and meta-analyses show that carbapenem use and medical devices are the leading risk factors for carbapenem-resistant Pseudomonas aeruginosa. Antimicrob Agents Chemother 2014; 58: 2626-2637.

6 Valentine VG, Gupta MR, Weill D, et al. Single-institution study evaluating the utility of surveillance bronchoscopy after lung transplantation. J Heart Lung Transplant 2009; 28: 14-20.

7 Glanville AR. The role of bronchoscopic surveillance monitoring in the care of lung transplant recipients. Semin Respir Crit Care Med 2006; 27: 480-491.

8 Kulkarni HS, Cherikh WS, Chambers DC, et al. Bronchiolitis obliterans syndrome-free survival after lung transplantation: an International Society for Heart and Lung Transplantation Thoracic Transplant Registry analysis. J Heart Lung Transplant 2019; 38: 5-16.

9 Glanville AR, Verleden GM, Todd JL, et al. Chronic lung allograft dysfunction: definition and update of restrictive allograft syndrome - A consensus report from the Pulmonary Council of the ISHLT. $J$ Heart Lung Transplant 2019; 38: 483-492.

10 Verleden GM, Glanville AR, Lease ED, et al. Chronic lung allograft dysfunction: definition, diagnostic criteria, and approaches to treatment - A consensus report from the Pulmonary Council of the ISHLT. J Heart Lung Transplant 2019; 38: 493-503.

11 Chambers DC, Cherikh WS, Harhay MO, et al. The International Thoracic Organ Transplant Registry of the International Society for Heart and Lung Transplantation: Thirty-sixth adult lung and heart-lung transplantation Report-2019; Focus theme: Donor and recipient size match. J Heart Lung Transplant 2019; 38: 1042-1055.

12 Levine DJ, Glanville AR, Aboyoun C, et al. Antibody-mediated rejection of the lung: a consensus report of the International Society for Heart and Lung Transplantation. J Heart Lung Transplant 2016; 35: 397-406.

13 Botha $\mathrm{P}$, Archer L, Anderson RL, et al. Pseudomonas aeruginosa colonization of the allograft after lung transplantation and the risk of bronchiolitis obliterans syndrome. Transplantation 2008; 85: 771-774.

14 Valentine VG, Gupta MR, Walker JE Jr, et al. Effect of etiology and timing of respiratory tract infections on development of bronchiolitis obliterans syndrome. J Heart Lung Transplant 2009; 28: 163-169.

15 Mainz JG, Hentschel J, Schien C, et al. Sinonasal persistence of Pseudomonas aeruginosa after lung transplantation. J Cyst Fibros 2012; 11: 158-161.

16 Beaume $\mathrm{M}$, Kohler $\mathrm{T}$, Greub $\mathrm{G}$, et al. Rapid adaptation drives invasion of airway donor microbiota by Pseudomonas after lung transplantation. Sci Rep 2017; 7: 40309.

17 Borthwick LA, Sunny SS, Oliphant V, et al. Pseudomonas aeruginosa accentuates epithelial-to-mesenchymal transition in the airway. Eur Respir J 2011; 37: 1237-1247.

18 Gregson AL, Wang X, Weigt SS, et al. Interaction between Pseudomonas and CXC chemokines increases risk of bronchiolitis obliterans syndrome and death in lung transplantation. Am J Respir Crit Care Med 2013; 187: 518-526.

19 Burkett A, Vandemheen KL, Giesbrecht-Lewis T, et al. Persistency of Pseudomonas aeruginosa in sputum cultures and clinical outcomes in adult patients with cystic fibrosis. Eur J Clin Microbiol Infect Dis 2012; 31: 1603-1610.

20 Mitchell AB, Oliver BG, Glanville AR. Translational aspects of the human respiratory virome. Am J Respir Crit Care Med 2016; 194: 1458-1464.

21 Dickson RP, Erb-Downward JR, Freeman CM, et al. Changes in the lung microbiome following lung transplantation include the emergence of two distinct Pseudomonas species with distinct clinical associations. PLoS One 2014; 9: e97214.

22 Bernasconi E, Pattaroni C, Koutsokera A, et al. Airway microbiota determines innate cell inflammatory or tissue remodeling profiles in lung transplantation. Am J Respir Crit Care Med 2016; 194: 1252-1263.

23 Borthwick LA, Suwara MI, Carnell SC, et al. Pseudomonas aeruginosa induced airway epithelial injury drives fibroblast activation: a mechanism in chronic lung allograft dysfunction. Am J Transplant 2016; 16: 1751-1765.

24 Smith S, Rowbotham NJ, Regan KH. Inhaled anti-pseudomonal antibiotics for long-term therapy in cystic fibrosis. Cochrane Database Syst Rev 2018; 3: CD001021. 